\title{
A Novel Microwave Tunable Band-Pass Filter Integrated Power Divider Based on Liquid Crystal
}

\author{
Yupeng Liu, ${ }^{1}$ Di Jiang, ${ }^{2}$ Lei Xia, ${ }^{1}$ and Ruimin $\mathrm{Xu}^{1}$ \\ ${ }^{1}$ School of Electronic Engineering, University of Electronic Science and Technology of China (UESTC), Chengdu, China \\ ${ }^{2}$ School of Communication and Information Engineering, University of Electronic Science and Technology of China (UESTC), \\ Chengdu, China \\ Correspondence should be addressed to Di Jiang; merryjiangdi@163.com
}

Received 18 September 2015; Revised 18 November 2015; Accepted 26 November 2015

Academic Editor: Ahmed A. Kishk

Copyright (C) 2015 Yupeng Liu et al. This is an open access article distributed under the Creative Commons Attribution License, which permits unrestricted use, distribution, and reproduction in any medium, provided the original work is properly cited.

\begin{abstract}
This paper proposes a novel microwave continuous adjustable band-pass filter integrated power divider based on nematic liquid crystals (LCs). The proposed power divider uses liquid crystal (LC) as the dielectric material. It can realize phase shift by changing the dielectric anisotropy, when biasing the high anisotropy nematic liquid crystal. It is mainly used in microwave frequencies. It has a large number of advantages compared to conventional filter integrated power divider, such as low loss, multifunction integration, continuous adjustable, miniaturization, low processing costs, low operating voltage, high phase shift, and convenient manufacture. Therefore, it has shown great potential for application.
\end{abstract}

\section{Introduction}

With the rapid development of modern wireless communication technology, it gives a higher request to the volume, performance, and integration of RF and microwave devices. And filter and power divider are two important passive components in the system. They sometimes take the form of cascade in the circuit. Therefore, with integration of two functions in one device, it is helpful to reduce connection loss and manufacturing cost and miniaturize the circuit. The current adjustable techniques mainly include diode switch, ferrite, ferroelectric substance, and RF MEMS. Yttrium iron garnet (YIG) has many advantages, but slow tuning speed and large volume are two of the biggest problems to limit its application [1]. Most indices of barium strontium titanate (BST) are in the middle level. But it has a poor linearity [2]. RF MEMS has better performances, but it has lesser tuning ratio and higher cost [3]. The tuning speed of diode is fast. But it has a poor linearity as well [4]. It is clear that the traditional microwave continuous adjustable filter and power divider fail to meet the developing requirements of technology. So it is the inevitable trend of development that the high performance microwave continuous adjustable band-pass filter integrated power divider is designed by new material, new technology, and new method [5].

Liquid crystal belongs to organic compounds. Its interior has long molecular structure. It is especially affected by the external environment. Especially in different concentration and temperature, it presents the orderly flow between liquid and solid. Thus, it has both the fluidity of liquid and the anisotropy of crystal. Liquid crystal is a fluid, whose configuration of director distribution or molecules is regular. Mainly, its optical properties, thermal properties, electrical properties, magnetic properties, and mechanical properties all present the crystal anisotropy. And these properties are closely related to liquid crystal molecules alignment. Also, liquid crystal is as liquid as common liquid. Although liquid crystal merges the main properties of crystal and liquid, it presents special magnetic and electric properties different from the ordinary crystal and liquid. Therefore, these properties of liquid crystal are widely used in different fields, particularly in the field of electromagnetic instruments. A lot of devices can be made of liquid crystal, such as liquid crystal sensitive print head, electronic aperture, phase diffraction grating, and spatial modulator. So, the applied 


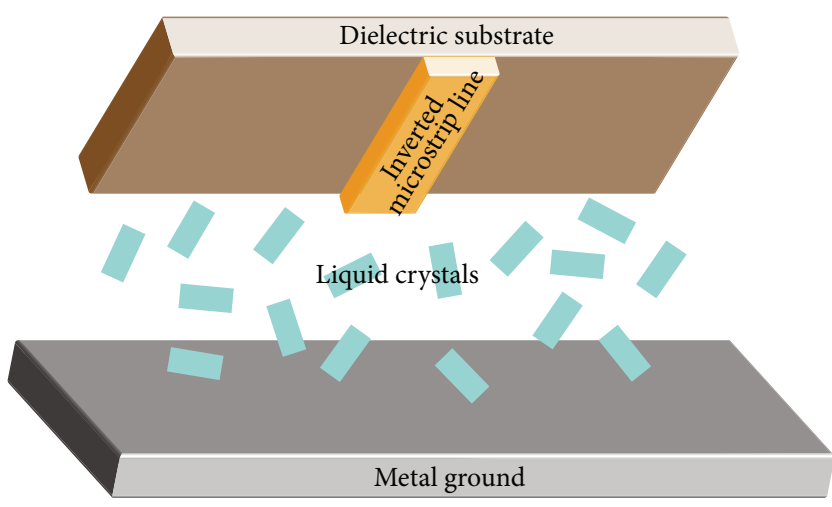

FIGURE 1: Idea structure of the novel circuit.

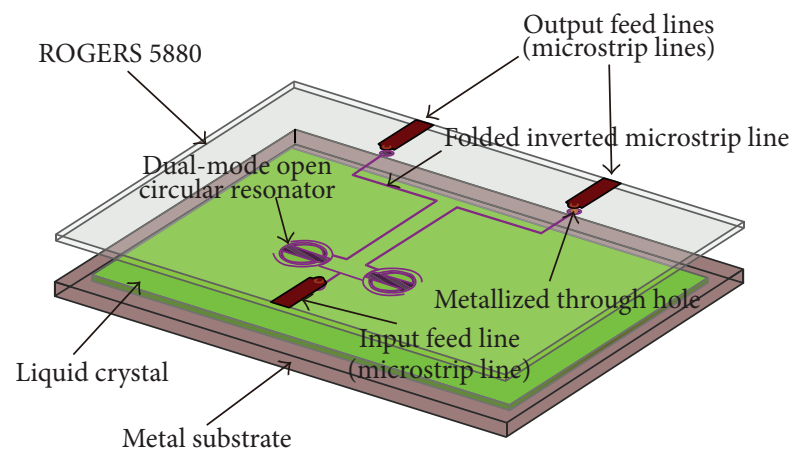

FIGURE 2: Schematic of continuous adjustable band-pass filter integrated power divider.

research of liquid crystal has become a research focus in the new interdiscipline [6].

In this paper, a novel microwave continuous adjustable band-pass filter integrated power divider, based on nematic liquid crystals with better performance for microelectromechanical system application, has been presented, and an idea structure of the novel liquid crystal circuit is shown in Figure 1.

\section{Filter Integrated Power Dividers Design}

As shown in Figure 2, this paper mainly uses folded inverted microstrip line structure. The inverted microstrip line has many advantages, such as simple structure, low dielectric loss, easy processing, and convenience in connecting with other microwave devices. In this design, the electric field in inverted microstrip line makes the higher efficiency on liquid crystal relative to the ordinary microstrip line.

The mentioned power divider is designed by ROGERS5880 with 1-layer substrate. The substrate has a thickness of $0.508 \mathrm{~mm}$ with a relative permittivity of 2.2 and loss tangent of 0.0009 . And its dimension is $31 \times 42 \mathrm{~mm}^{2}$. This material has high dielectric consistency, low dielectric loss, and certain hardness. And it is mainly for resonators for power distribution of band-pass filter integrated power divider and input/output feed lines. Resonators for power distribution of band-pass filter integrated power divider

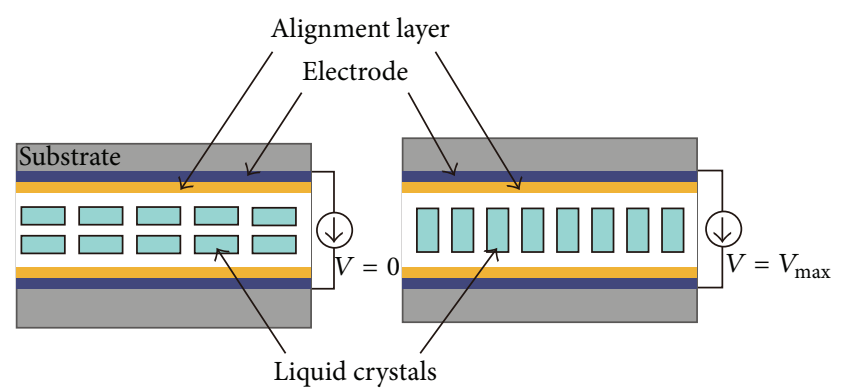

FIGURE 3: Working principle diagram of the nematic liquid crystals.

include dual-mode open circular resonators, T-junction for power divider, and folded transmission lines on two ports. There are two dual-mode open circular resonators, which are located in two separate branches of power divider. These resonators are etched in the back of the upper substrate. Two folded transmission lines on ports are also etched in the back of the upper substrate for matching these ports. Input/output feed lines are etched in the front of the upper substrate, which are connected to those dual-mode open circular resonators metallized through holes. And they are used to transmit microwave signals and feed modulation voltage signal. Etching a $0.1 \mathrm{~mm}$ thickness of slot on the upper surface of the metal substrate is used for carrying liquid crystals; that is, the thickness of LC is $0.1 \mathrm{~mm}$. We use anisotropic conductive film (ACF) to connect the proposed ROGERS5880 substrate and the metal substrate for package stacking. Then we trickled the nematic liquid crystals into the slot by filling plug, using capillary attraction [7-9].

As shown in Figure 3, when the nematic liquid crystals work without bias voltage $(V=0)$, the long axes of the liquid crystal molecules are parallel to each other and parallel to the ROGERS5880 substrate. At this point, the measured dielectric constant is $\varepsilon_{\perp}$. When applying an electric field $E$ between the metallic ground plane and the inverted microstrip line with bias voltage $\left(V=V_{\max }\right)$, the orientation of liquid crystal molecules will gradually turn to the direction of the electric field. When the bias voltage is high enough and achieves the saturation voltage of liquid crystals, the polarization of the nematic liquid crystals reaches saturation. At this point, the measured dielectric constant is $\varepsilon_{/ /}$. And the dielectric constant stops changing over bias voltage $V_{\max }$. Thus, it realizes to modulate dielectric constant. For a certain physical size of the resonator, its electric length is also modulated by the external voltage. By this way, the resonant frequency of the proposed power divider changes accordingly. And finally it implements continuous tuning for band-pass $[10,11]$. These optimization works are managed by using commercial 3D electromagnetic software HFSS.

\section{Results and Discussion}

To verify the model, a novel adjustable filter integrated power divider is simulated, fabricated, and tested at room temperature $25^{\circ} \mathrm{C}$. The photograph of the fabricated device is shown in Figures 4 and 5. As the front content argues, Figure 6 


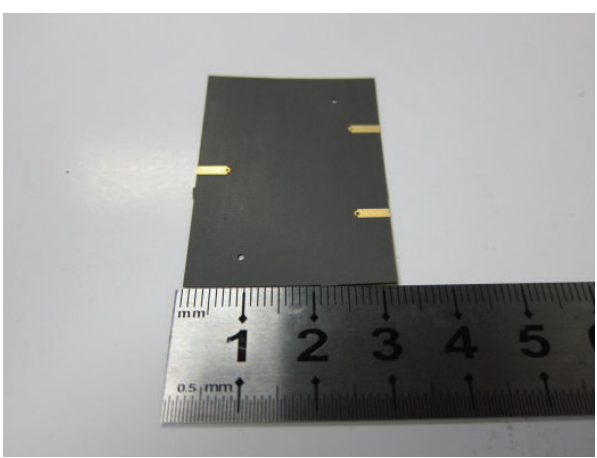

(a)

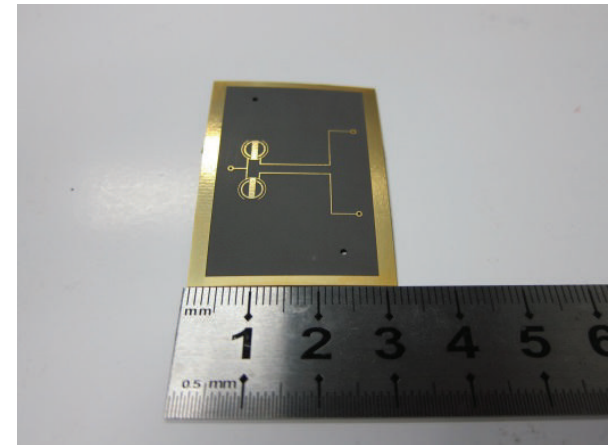

(b)

FIGURE 4: Photograph of the proposed ROGERS5880 substrate. (a) Left view; (b) right view.
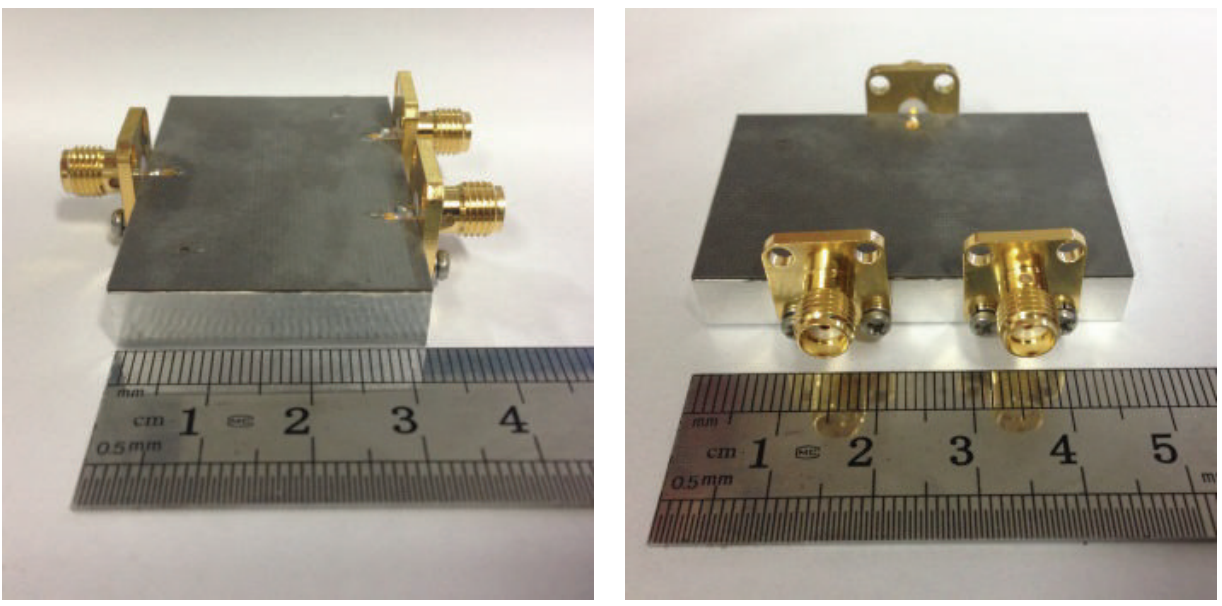

FIGURE 5: Photograph of the proposed novel filter integrated power divider.

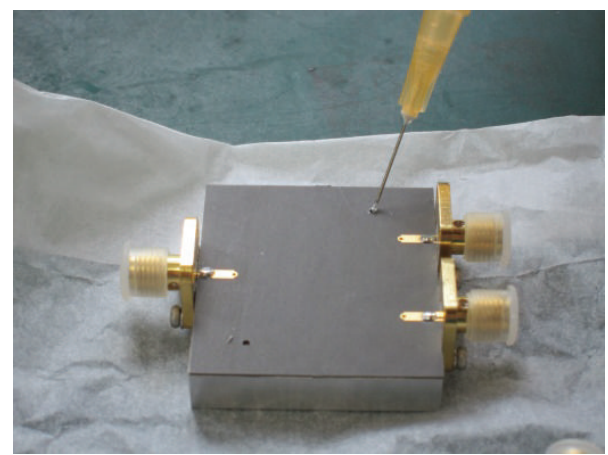

FIGURE 6: The LC filling method.

shows that we trickled the nematic liquid crystals into the slot by filling plug, using capillary attraction. And Figure 7 shows the test environment. The peak voltage provided by function/arbitrary waveform generator, ranges from 0 to $20 \mathrm{~V}$. And the corresponding waveform used for driving the LC material is $1 \mathrm{kHz}$ square signal.

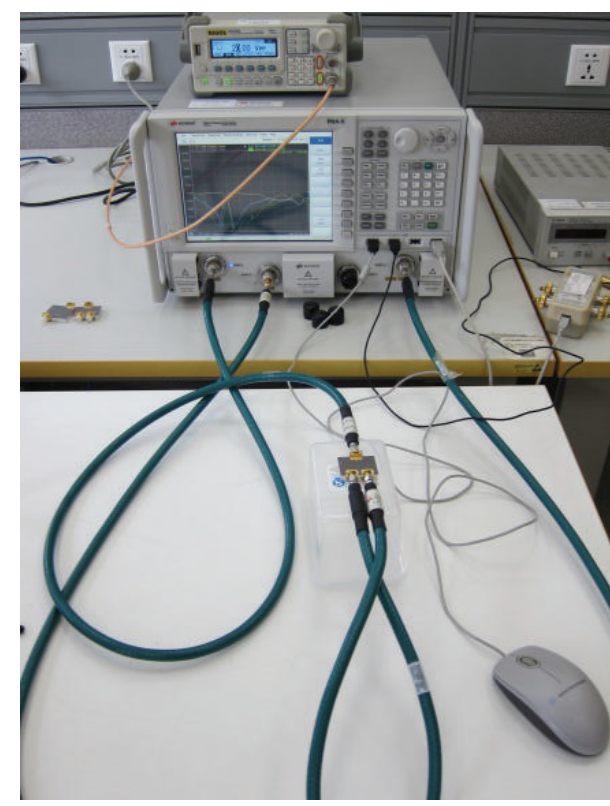

FIGURE 7: The testing platform. 


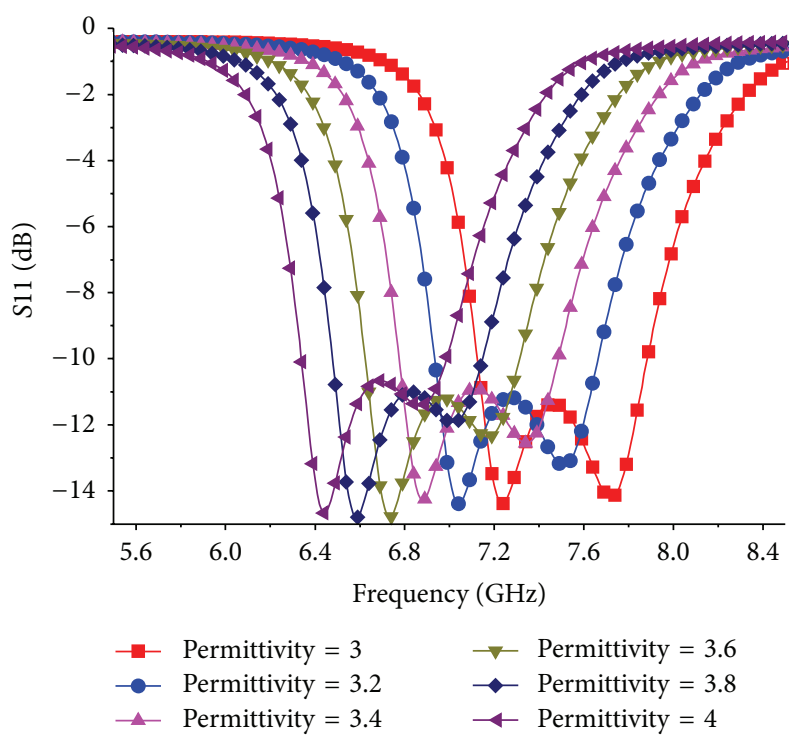

(a)

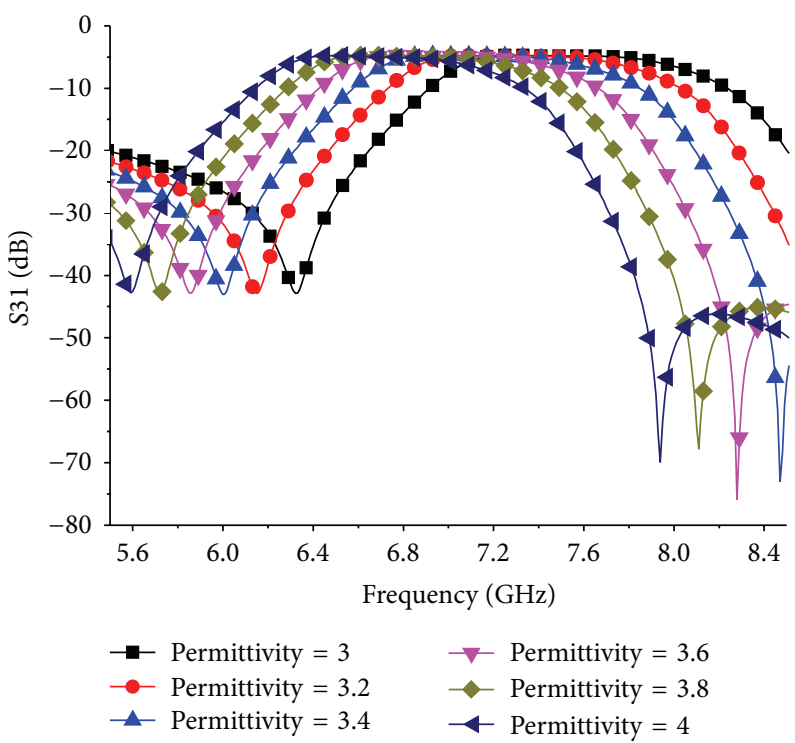

(c)

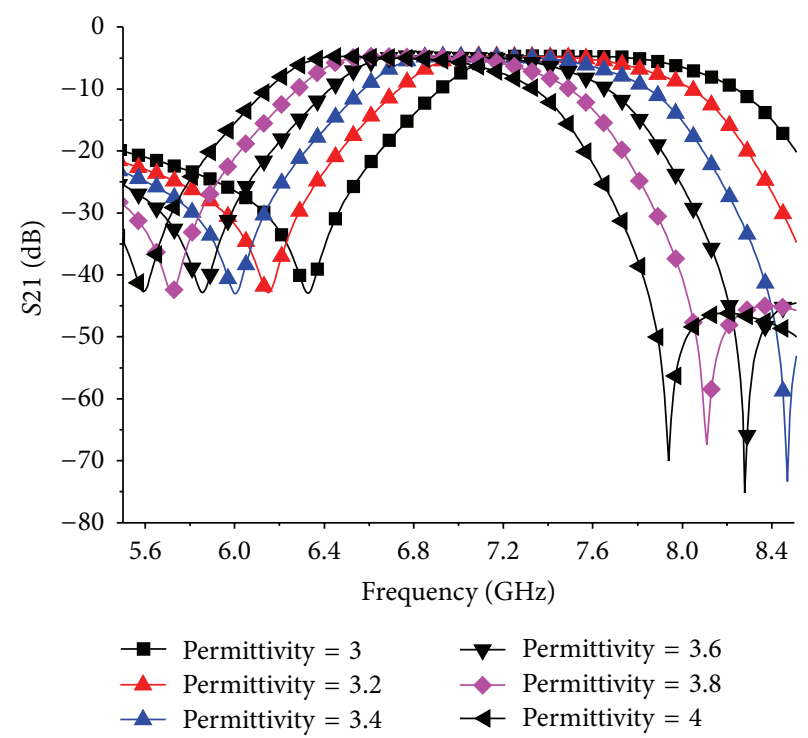

(b)

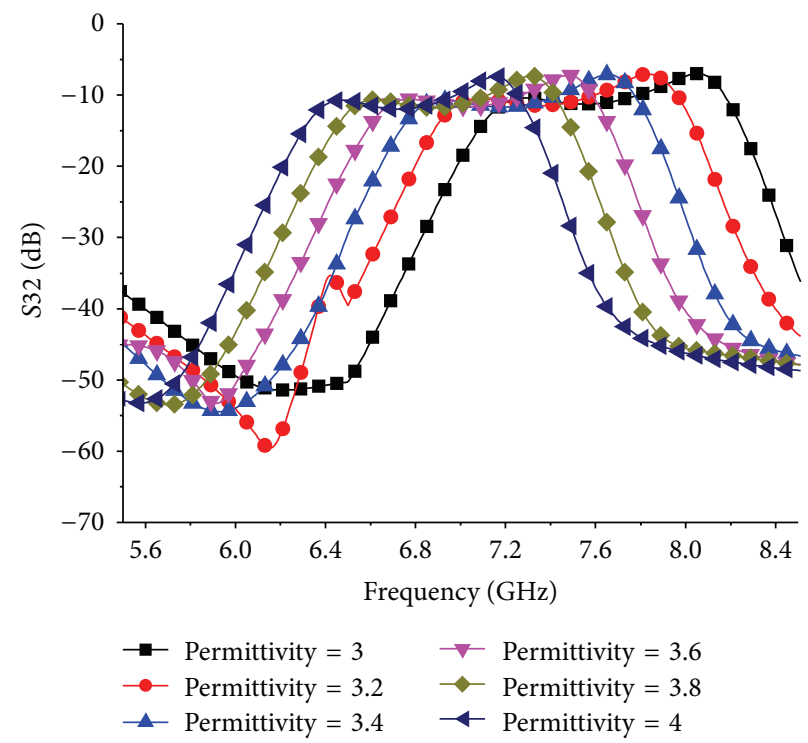

(d)

FIGURE 8: Simulated S-parameters with different LC permittivities. (a) S11; (b) S21; (c) S31; (d) S32.

The relevant simulated and measured scattering parameters of the prototype are shown in Figures 8 and 9, respectively. Figure 8 shows that it makes the center frequency to achieve $1 \mathrm{GHz}$ frequency offset. The dual-mode open circular resonators can determine the central frequency of the power divider. For this structure, the central frequency is $7 \mathrm{GHz}$. The simulation results show a filter bandwidth of $1 \mathrm{GHz}$. The return loss at the input port (S11) is better than $10 \mathrm{~dB}$ and the insertion losses (S21 and S31) are less than $4.7 \mathrm{~dB}$ over the band as shown in Figures 8(a), 8(b), and 8(c). And the isolation between two output ports (S32) is better than $10 \mathrm{~dB}$ all over the band as shown in Figure 8(d).

According to the electric field analysis of simulation model, it is clear that the proposed filter power divider realizes the equal power allocation in the corresponding frequency range.
Finally, the proposed model is validated by the good agreement between simulation results and test data in Figures 8 and 9.

However, due to fabrication tolerances and the position displacement of SMA adaptors, the measured insertion losses (S21 and S31) of the fabricated model are higher than the simulated results.

In this design, as an ideal homogeneous material, the LC has many advantages, such as high purity, performance good consistency, and high surface finish. But in this practice, some uncertainties of filling content affect dielectric constant, which lead to less obvious frequency offset. In addition, mismachining tolerance of inverted microstrip lines affects the center frequency. Besides, the $0.508 \mathrm{~mm}$ thick ROGERS5880 substrate is prone to be deformation caused by machining process and differences between the internal 


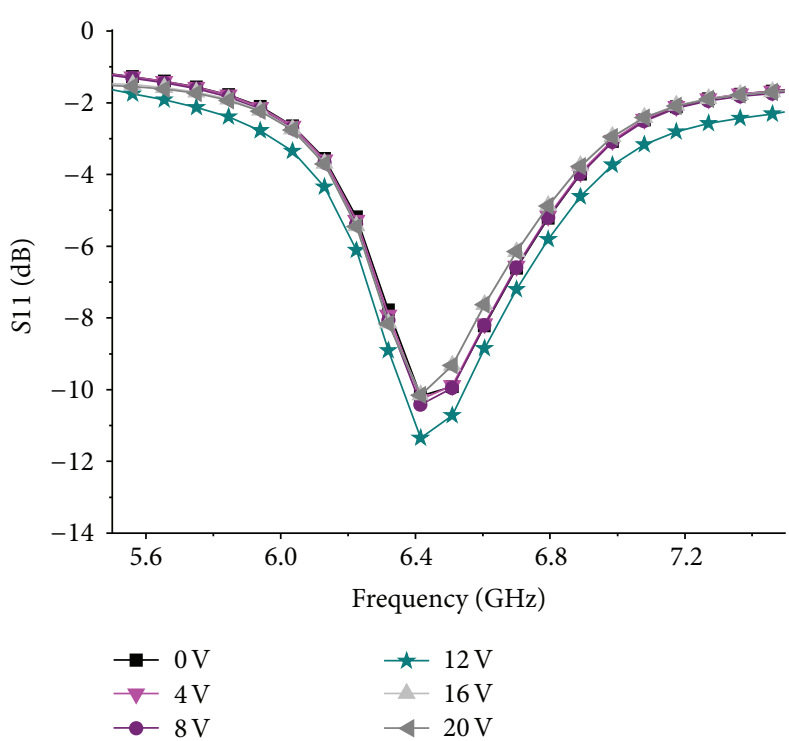

(a)

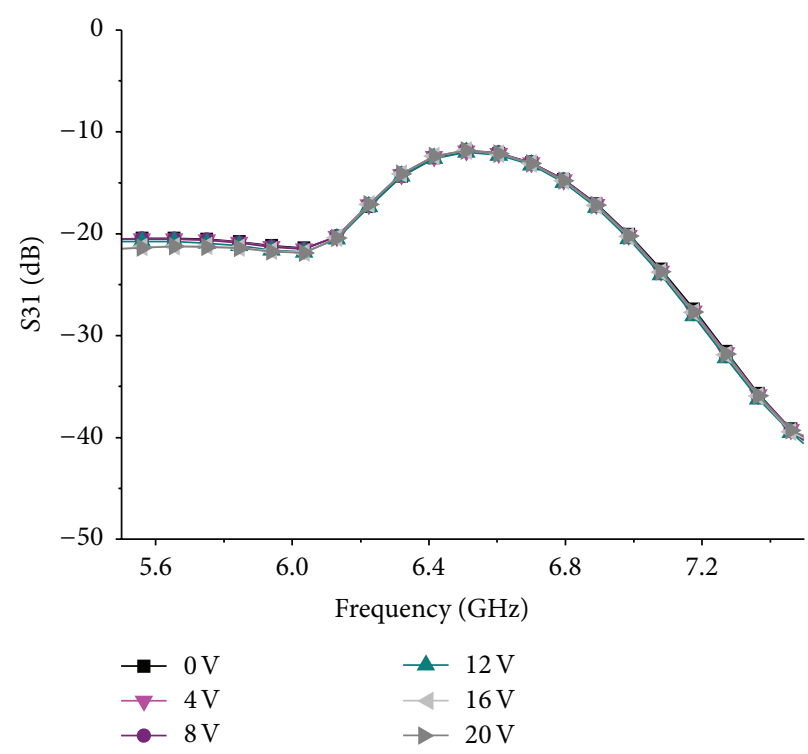

(c)

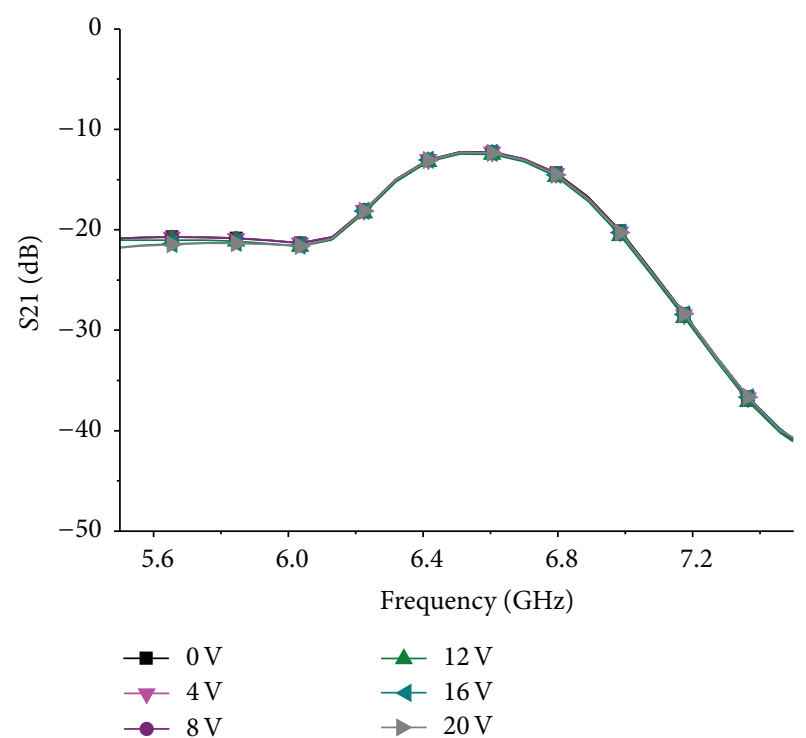

(b)

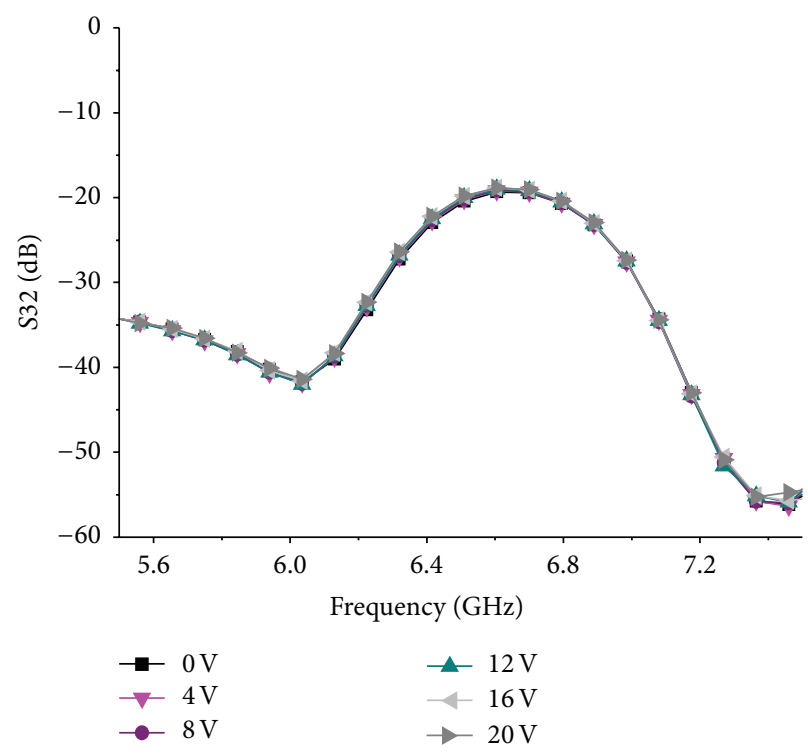

(d)

FIGURE 9: Measured frequency response under application of various peak voltages. (a) S11; (b) S21; (c) S31; (d) S32.

pressure and the external pressure after assembly, which also leads to nonuniform filling to liquid crystal and less obvious frequency offset.

\section{Conclusions}

In this letter, a novel microwave continuous adjustable band-pass filter integrated power divider, based on nematic liquid crystals, is proposed. Compared with the traditional power dividers, the proposed power divider has obvious band-pass and good isolation. Meanwhile, it provides a new design method of the microwave continuous adjustable band-pass filter integrated power divider for solving existing problems, such as the narrow technical tuning range, the poor linearity, high cost, and the complex control logic.
Therefore, the results of this work are useful for microwave system.

\section{Conflict of Interests}

The authors declare that there is no conflict of interests regarding the publication of this paper.

\section{Acknowledgments}

This work was supported by National Natural Science Foundation of China (Grant no. 61501091), the Science and Technology Support Plan of Sichuan Province (2015RZ0012), the Central University Basic Research Funding (ZYGX2014J003), and Shanghai Aerospace Science and Technology Innovation 
Fund Project, Guangxi Key Laboratory of Wireless Wideband Communication and Signal Processing.

\section{References}

[1] M. A. Popov, I. V. Zavislyak, and G. Srinivasan, "Yttrium iron garnet magnetically tunable magnetodielectric resonator for millimeter and submillimeter wavelength," in Proceedings of the 21th International Crimean Conference Microwave and Telecommunication Technology (CriMiCo '11), pp. 678-679, IEEE, Sevastopol, Ukraine, September 2011.

[2] D. Kuylenstierna, A. Vorobiev, and S. Gevorgian, "40 GHz lumped element tunable bandpass filters with transmission zeros based on thin $\mathrm{Ba}_{0.25} \mathrm{Sr}_{0.75} \mathrm{TiO}_{3}$ (BST) film varactors," in Proceedings of the Topical Meeting on Silicon Monolithic Integrated Circuits in RF Systems, pp. 342-345, San Diego, Calif, USA, January 2006.

[3] A. Takacs, D. Neculoiu, D. Vasilache et al., "Tunable bandstop and bandpass MEMS filters for millimeter wave applications," in Proceedings of the 38th European Microwave Conference (EuMC '08), pp. 591-594, Amsterdam, The Netherlands, October 2008.

[4] J. Jian Xu, X.-P. Liang, and K. Shamsaifar, "Full wave analysis and design of RF tunable filters," in Proceedings of the IEEE MTT-S International Microwave Symposium Digest, pp. 14491452, IEEE, Phoenix, Ariz, USA, May 2001.

[5] M. A. El-Tanani and G. M. Rebeiz, "A two-pole two-zero tunable filter with improved linearity," IEEE Transactions on Microwave Theory and Techniques, vol. 57, no. 4, pp. 830-839, 2009.

[6] M. Yazdanpanahi, S. Bulja, D. Mirshekar-Syahkal, R. James, S. E. Day, and F. A. Fernandez, "Liquid-crystal-based mm-wave tunable resonator," in Proceedings of the IEEE European Microwave Conference (EuMC '10), pp. 1233-1236, Paris, France, September 2010.

[7] V. Urruchi, C. Marcos, J. Torrecilla, J. M. Sánchez-Pena, and K. Garbat, "Note: tunable notch filter based on liquid crystal technology for microwave applications," Review of Scientific Instruments, vol. 84, no. 2, Article ID 026102, 2013.

[8] D. Jiang, H. Chen, X. Li, Z. He, and M. Shen, "A novel tunable microwave filter integrated power dividers using liquid crystal," Microwave and Optical Technology Letters, vol. 57, no. 11, pp. 2539-2543, 2015.

[9] R. Follmann, D. Kother, M. A. Campo et al., "Liquida-sky-a tunable liquid crystal filter for space applications," in Proceedings of the IEEE-APS Topical Conference on Antennas and Propagation in Wireless Communications (APWC '13), pp. 90-93, Torino, Italy, September 2013.

[10] S. Mueller, A. Penirschke, C. Damm et al., "Broad-band microwave characterization of liquid crystals using a temperaturecontrolled coaxial transmission line," IEEE Transactions on Microwave Theory and Techniques, vol. 53, no. 6, pp. 1937-1945, 2005.

[11] A. Penirscjke, S. Muller, P. Scheele et al., "Cavity perturbation method for characterization of liquid crystals up to $35 \mathrm{GHz}$," in Proceedings of the 34th European Microwave Conference, vol. 2, pp. 545-548, IEEE, Amsterdam, The Netherlands, October 2004. 

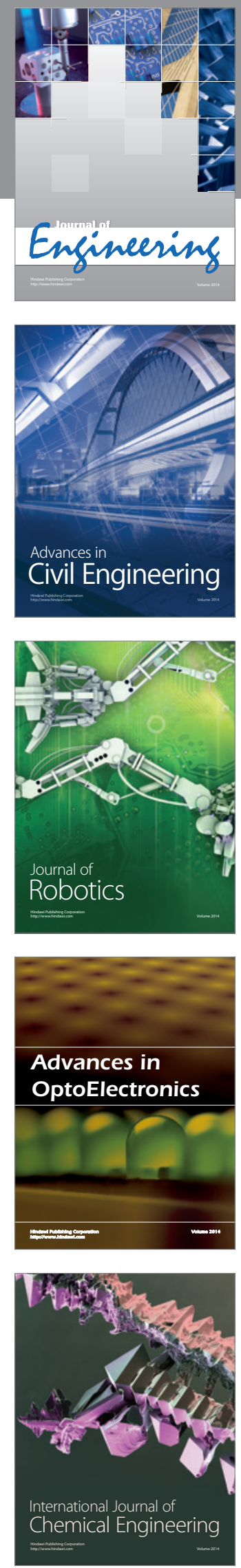

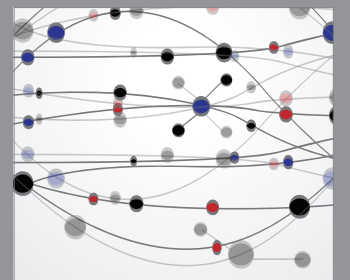

The Scientific World Journal
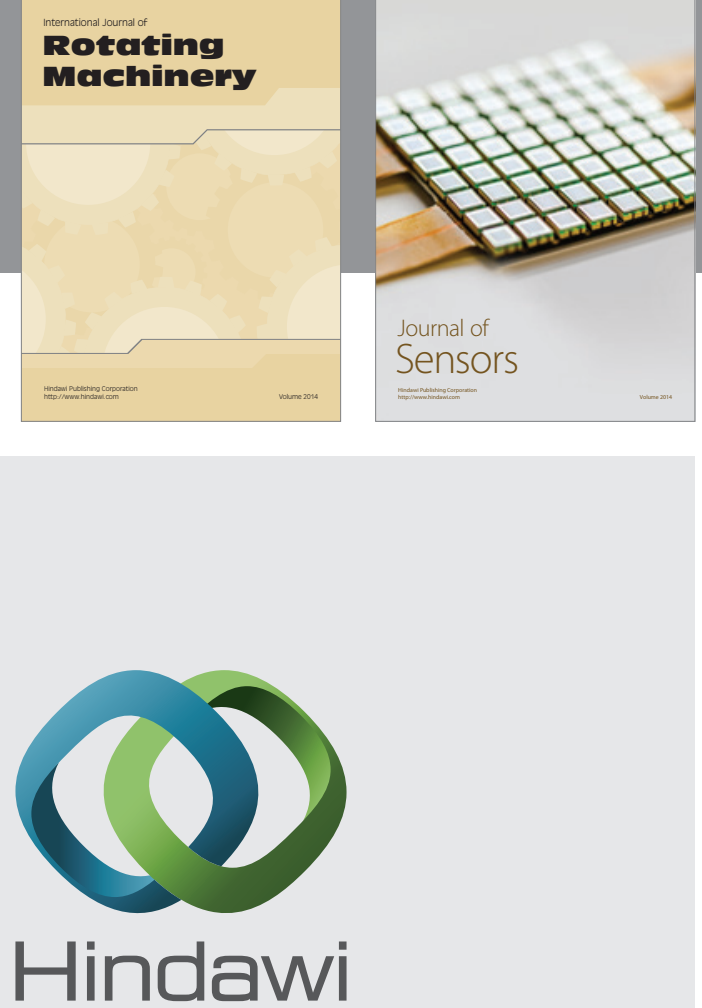

Submit your manuscripts at http://www.hindawi.com
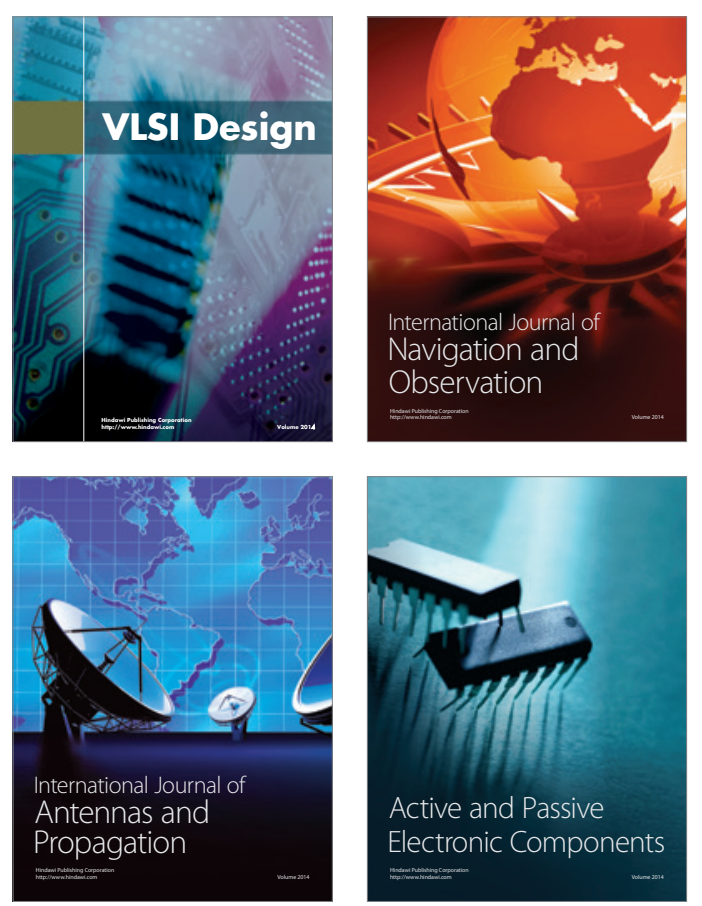
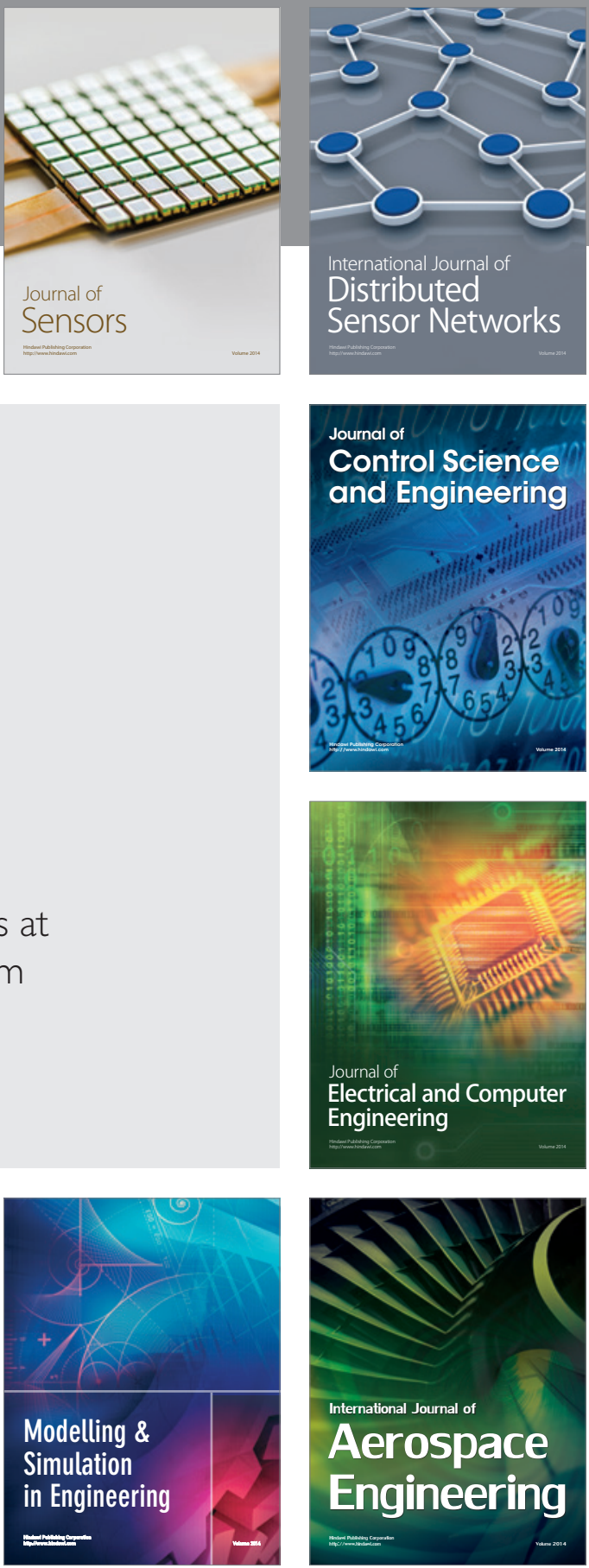

Journal of

Control Science

and Engineering
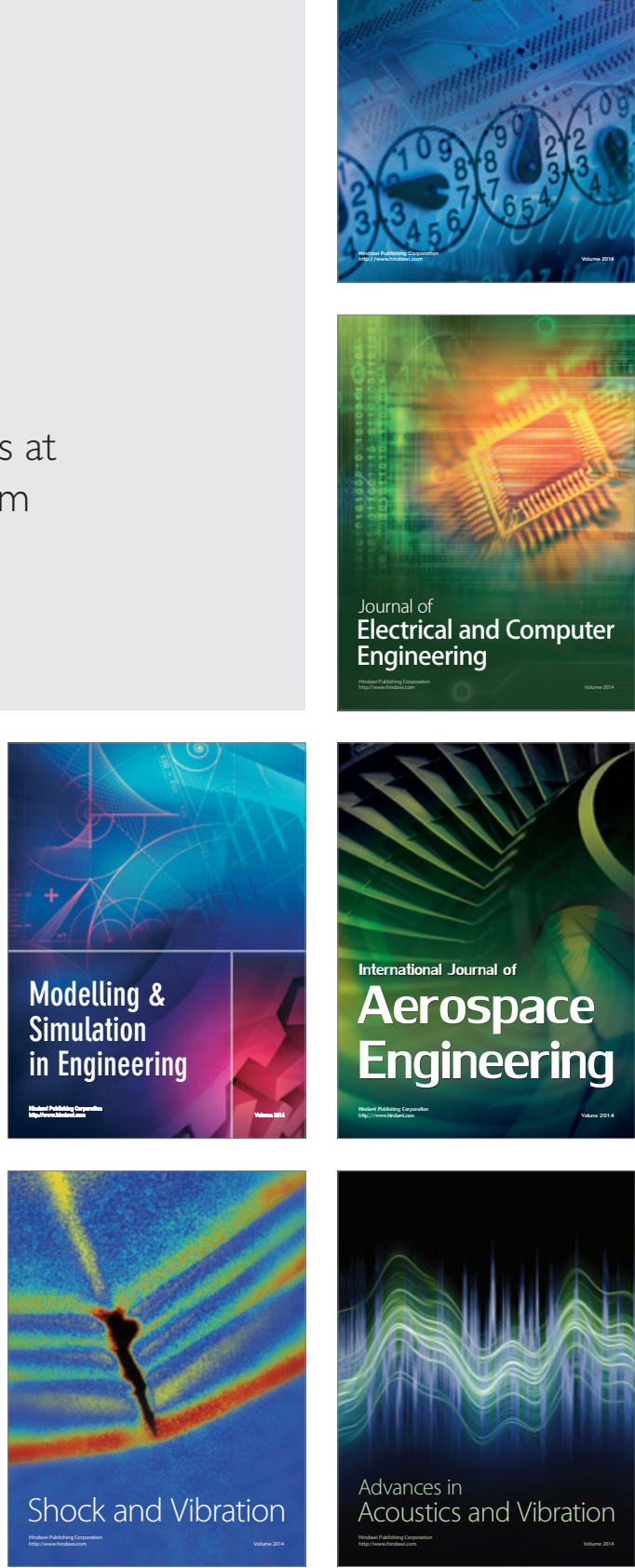